\title{
Abrogation of PIK3CA or PIK3R1 reduces proliferation, migration, and invasion in glioblastoma multiforme cells
}

\author{
Genevieve L. Weber ${ }^{1}$, Marie-Odile Parat ${ }^{2}$, Zev A. Binder ${ }^{1}$, Gary L. Gallia ${ }^{1}$, Gregory \\ J. Riggins ${ }^{1}$ \\ ${ }^{1}$ Ludwig Collaborative Laboratory, Department of Neurosurgery, Johns Hopkins University School of Medicine, Baltimore, \\ MD \\ 2 School of Pharmacy, University of Queensland, Australia \\ Correspondence to: Gregory J. Riggins, email: griggin $1 @ j h m i . e d u$
}

Keywords: glioblastoma, PIK3CA, PIK3R1, invasion, PI3K, pathway analysis

Received: October 27, 2011, Accepted: October 28, 2011, Published: November 5, 2011

Copyright: ( ) Weber et al. This is an open-access article distributed under the terms of the Creative Commons Attribution License, which permits unrestricted use, distribution, and reproduction in any medium, provided the original author and source are credited.

ABSTRACT:

\begin{abstract}
Glioblastoma multiforme (GBM) is a highly invasive and deadly brain tumor. Tumor cell invasion makes complete surgical resection impossible and reduces the efficacy of other therapies. Genome-wide analyses of mutations, copy-number changes, and expression patterns have provided new insights into genetic abnormalities common in GBM. We analyzed published data and identified the invasion and motility pathways most frequently altered in GBM. These were most notably the focal ad hesion and integrin signaling, and extracellular matrix interactions pathways. We mapped alterations in each of these pathways and found that they included the catalytic PIK3CA and regulatory PIK3R1 subunit genes of the class IA PI3K. Knockdown of either of these genes separately in GBM cell lines by lentiviral-mediated shRNA expression resulted in decreased proliferation, migration, and invasion in all lines tested. FAK activity was reduced by knockdown of either PIK3CA or PIK3R1, and MMP2 levels were reduced by knockdown of PIK3R1. We conclude that PIK3R1, like PIK3CA, is a potential therapeutic target in GBM and that it also influences tumor cell growth and motility.
\end{abstract}

\section{INTRODUCTION}

Glioblastoma multiforme is the most common primary brain malignancy, with a current median survival of approximately 15 months in patients with newly diagnosed disease following treatment with surgery, chemotherapy and radiotherapy [1]. The invasive nature of GBM has been frequently implicated as a key feature of GBM's resistance to therapy [2-5]. Targeting pathways involved in the invasive phenotype of the tumor could be an important component of successful treatment in combination with strategies that more directly target the cancer cells.

The sequencing of 20,661 gene coding regions in multiple glioblastoma tumors was a significant milestone in understanding the genetic basis of GBM [6]. In this analysis, Parsons, et al utilized automated sequencing approaches and Illumina SNP arrays to identify mutations and copy number changes, respectively, in genes in 22 glioblastomas [6]. Genes found to harbor alterations in at least 2/22 tumors, and which had mutation frequencies of $>10$ mutations per $\mathrm{Mb}$ of DNA sequenced, were subsequently re-sequenced in an additional 83 tumors as validation. This sequencing of 22 GBM exomes, along with detection of genomic amplifications and homozygous deletions, likely identifies the majority of the mutated genes driving tumor formation and progression in glioblastoma.

One challenge has been to identify the genetic alterations that occur during tumor development that are functionally involved in driving tumor formation and progression. Carter et al developed the Cancer-specific High-throughput Annotation of Somatic Mutations (CHASM) program to systematically identify mutations in tumors that are likely to drive the cancer phenotype [7]. The CHASM program utilizes 49 predictive features to estimate the likelihood that a specific mutation in question is a driver, rather than a passenger mutation [7]. This group applied CHASM to a list of 607 missense mutations found by Parsons et al in GBM [6, 7]. The 
result was a stringent list of 24 mutations ( $8 \%$ of the total mutation list) that have a much higher probability of being functionally involved in GBM pathogenesis, and included alterations found in the PTEN, TP53, IDH1, and PIK3CA genes, among others.

The mutations of functional consequence in GBM are responsible for many aspects of its malignant phenotype, including cellular invasion. Glioblastoma cells exhibit significantly increased motility and invasive potential as compared to many brain tumors of lower grade $[8,9]$. Tumor cell invasion results in an inability to cure the tumor via surgery alone, and invading tumor cells are more resistant to apoptosis, radiation and certain chemotherapies [5, 8-10]. It is likely that anti-invasion/ motility therapy might render these cells more susceptible to apoptosis-based chemotherapeutics.

Glioblastoma cell invasion likely occurs through multiple mechanisms, beginning with the degradation of surrounding matrix proteins by proteases and proteinases in order to create a cavity through which a tumor cell can migrate $[4,9,11]$. Invading cells must detach from neighboring cells and matrix components in order to become motile $[9,11]$. Cells can then move through healthy brain tissue in a receptor-mediated fashion that requires receptor turnover, including the formation and degradation of focal adhesions, and cytoskeletal rearrangements [9, 12]. Cell attachments, along with receptor and focal adhesion turnover and cytoskeleton changes, are controlled in part by complex interactions between integrins, receptor tyrosine kinases, and pathways such as focal adhesion kinase (FAK) and phosphatidylinositol 3-kinase (PI3K) signaling [12-16]. Several ECM components themselves, including laminin and fibronectin, have been shown to be overexpressed in tumors, and downregulation of these components reduces invasion and migration of glioblastoma cells [17-21]. The rate of proliferation of invading tumor cells is often significantly decreased when compared to cells in the main tumor mass $[8,9]$. Targeting both proliferating and migrating cells is likely essential for an effective therapy.

The PI3K cascade is an important pathway known to be involved in proliferation, invasion, and migration in cancer [22-24]. Class IA PI3Ks are heterodimers of a p85 regulatory subunit and a p110 catalytic subunit [25, 26]. Three catalytic subunits exist, and are designated $\mathrm{p} 110 \alpha, \mathrm{p} 110 \beta$, and $\mathrm{p} 110 \delta$. Five class IA PI3K regulatory subunits include $\mathrm{p} 85 \alpha, \mathrm{p} 85 \beta, \mathrm{p} 50 \alpha, \mathrm{p} 55 \alpha$, and $\mathrm{p} 55 \gamma$. The $\mathrm{p} 85 \alpha$ regulatory and $\mathrm{p} 110 \alpha$ catalytic subunits are the most highly expressed and form heterodimers most commonly [26]. Upon localization of the heterodimer to the plasma membrane via binding of Src homology 2 (SH2) domains on the regulatory subunit to activated receptor tyrosine kinases, the regulatory subunit releases its inhibitory control of the catalytic subunit, and the catalytic subunit comes in close contact with its lipid substrates. The catalytic subunit phosphorylates phosphatidylinositol 4,5-bisphosphate (PIP2), converting it to phosphatidylinositol 3,4,5-triphosphate (PIP3). PIP3 can activate several downstream signaling cascades, including the Akt and mTOR pathways, which are involved in proliferation and cell survival. PTEN activity converts PIP3 to PIP2, thus regulating the level of activation of these downstream pathways. Constitutive activation of the Akt and/or mTOR pathways and loss of function of PTEN can each contribute to tumor progression $[27,28]$.

The PIK3CA gene, which encodes the class IA PI3K catalytic subunit $\mathrm{p} 110 \alpha$, has been found to harbor mutations in several cancers [29-31]. Approximately 80\% of p $110 \alpha$ mutations cluster in "hot spots" in the helical (E542K and E545K in exon 9) and kinase (H1047R in exon 20) domains, and were considered to be likely drivers of cancer formation and/or progression by Parsons et al and Carter et al $[6,7,29]$. However, the majority of mutations discovered in PIK3CA, regardless of their location, have been shown to constitutively activate the catalytic activity of $\mathrm{p} 110 \alpha$ [32].

The PIK3R1 gene, which encodes the p85a, p55 $\alpha$, and $\mathrm{p} 50 \alpha$ class IA PI3K regulatory subunits, was found to be mutated in glioblastoma tumors by Parsons et al and amplified in GBMs in multiple studies $[6,33]$. PIK3R 1 is also mutated in other human cancers, including colorectal cancer, breast cancer, ovarian cancer, and endometrial cancer [34-36].

In many cases, PIK3R1 mutations are thought to act through the catalytic subunit $[34,37,38]$. p $85 \alpha$ inhibits the activity of the catalytic subunit by binding of its $\mathrm{N}$-terminal SH2 domain to the N-terminus of the catalytic subunit [39]. Interaction with a regulatory subunit is also important for the stability of the catalytic subunit, which may degrade more rapidly in an unbound state [25]. A significant number of mutations documented in PIK3RI are located in the inter-SH2 or N-terminal SH2 domain of $\mathrm{p} 85 \alpha$, which may result in a regulatory subunit that no longer inhibits activity, but still maintains stability, of the catalytic subunit $[40,41]$. Since $\mathrm{p} 85 \alpha$ regulates the class IA PI3K catalytic subunit, mutations in PIK3R1 may potentially activate invasion.

Our hypothesis for this work is that there are common mutations that activate a defined set of pathways that are responsible for the invasive properties in glioblastoma cells. We performed an in-depth informatics analysis to search for invasion and migration-related pathways that are enriched with mutations in GBM. With the recent availability of a well defined set of mutations responsible for GBM, we hope to determine the likely mutational basis responsible for at least part of GBM's invasive phenotype. We mapped many of the GBM mutations to pathways that may influence the invasive or migratory phenotype of this tumor, including focal adhesion signaling, integrin signaling, and extracellular component and receptor signaling. We show, as have multiple lines of pre-existing evidence that the PI3K signaling cascade is involved in 
each of these pathways and is likely to play an important role in both the proliferative and migratory/invasive phenotypes observed in GBM cells. shRNA-mediated knockdown of either the PIK3CA or PIK3R1 gene resulted in reduced proliferation, migration, and invasion in glioblastoma cell lines, and in the case of the PIK3RI gene these effects are independent of the Akt pathway. Our studies suggest for the first time that PIK3R1 may play an important role in the invasive and migratory capabilities of GBM cells, and that the p $85 \alpha$ product of this gene may be an effective anti-invasion target.

\section{RESULTS}

\section{Invasion and migration-related pathways are mutated in GBM}

Using GBM sequencing and genomic copy number data, we sought statistically significant invasion and motility-related pathways enriched for genes mutated in GBM. A similar analysis was performed using gene expression data from the same tumors sequenced for mutation.

Our analyses included a comprehensive list of 703 genes mutated in GBM (Table S2), and a smaller more stringent list of 59 high probability driver mutations (Table S3) developed from the combined findings of Parsons et al [6], Carter et al [7], and Rao et al [33]. Transcript counts produced by next generation sequencing, a variation of Serial Analysis of Gene Expression (SAGE), were analyzed from Parsons et al [6]. This gene expression data covered 20,619 curated genes in 16 human tumors. Mutation data was analyzed using Ingenuity Pathway Analysis and Partek Genomics Suite, while SAGE data was studied using Spotfire DecisionSite. The use of Partek and Ingenuity simultaneously increases the validity and significance of our analysis, given that these programs produce results based upon algorithms that are complementary, but do not overlap.

Using the expanded and stringent mutated gene lists, we searched for enriched ontologies, based upon molecular function, cellular component, and biological process as defined by the Gene Ontology Consortium, using Partek Genomics Suite. Additionaly, we searched for enriched canonical pathways using Ingenuity Pathway Analysis, which ranks genes based on their function rather than inclusion within an ontological group. Both programs generated lists of pathways altered in GBM, each of which was assigned statistical significance ( $p$-value greater than or equal to 0.5) (Tables S4-S7). We found that many of the statistically significant frequently mutated pathways were involved directly in the invasive or migrational phenotype in GBM (Tables S8, S9). Within these pathways, we also analyzed SAGE expression data to determine the presence of genes up- or down-regulated by 5-fold or greater. We mapped alterations in several of these enriched pathways, including focal adhesion signaling (Fig. S1), integrin signaling (Fig. S2), and extracellular matrix interactions (Fig. S3).

\section{Class IA PI3K plays a role in most invasion and migration-related pathways in GBM}

In studying the genes involved in the mutationenriched invasion and migration-related pathways, we found that the class IA PI3K subunit genes PIK3CA and $P I K 3 R 1$ were central signal transducers in essentially all of the pathways studied (Fig. 1, S1, S2). PIK3CA is mutated in approximately $10 \%$ of GBMs and, $P I K 3 R 1$ is mutated in approximately $8 \%[29,31,35,42]$. The role of $P I K 3 C A$ in tumor cell proliferation is well-studied as compared to its role in invasion. Examination of the original gene list (703 mutated genes) and the stringent gene list (59 likely driver mutations) produced results that were typically in agreement (Tables S4-S9). Results (p-values) will be discussed in terms of the stringent gene list analysis, due to the higher likelihood that these mutations are drivers of GBM formation. When this list was analyzed in Ingenuity Pathway Analysis, PIK3CA and PIK3R1 were found to be involved in several important motility pathways, including focal adhesion $(\mathrm{p}<0.0001)$, integrin $(\mathrm{p}=$ $0.0004)$, Rac ( $\mathrm{p}=0.0046)$, and axonal guidance signaling $(\mathrm{p}=0.0065)$. Similarly, when this list was analyzed in Partek Genomics Suite, both subunits were found to be involved in regulation of cell adhesion $(\mathrm{p}<0.0001)$, cell motion $(p<0.0001)$, focal adhesion formation $(p<0.0001)$, and the integrin-mediated signaling pathway $(\mathrm{p}=0.0019)$. The results of analyses in Partek and Ingenuity, which use complementary algorithms but do not overlap, were in agreement in terms of the significance of the invasion and migration-related pathways that are mutated/aberrantly expressed in GBM, and in the involvement of PIK3CA and PIK3R1 in these pathways. The PI3K cascade itself was found to be statistically significantly altered in GBM by both the Ingenuity $(p<0.0001)$ and Partek programs $(\mathrm{p}<0.0001)$.

\section{The SKMG26, HCT116, and 081024 cell lines harbor PIK3CA mutations}

In determining the mutational status of the $P I K 3 C A$ gene in our cell lines, we sequenced the 10 most commonly mutated exons $(1,2,4,5,7,9,12,13,18,20)[6,29,31]$. One glioblastoma line, SKMG26, and one colorectal cancer line, HCT116, have been previously sequenced. SKMG26 and HCT116 lines each bear a single somatic mutation in the kinase domain of the catalytic subunit (exon 20) [29, 31]. These mutations induce H1047Y and H1047R amino acid changes, respectively [29, 31]. In SKMG26 


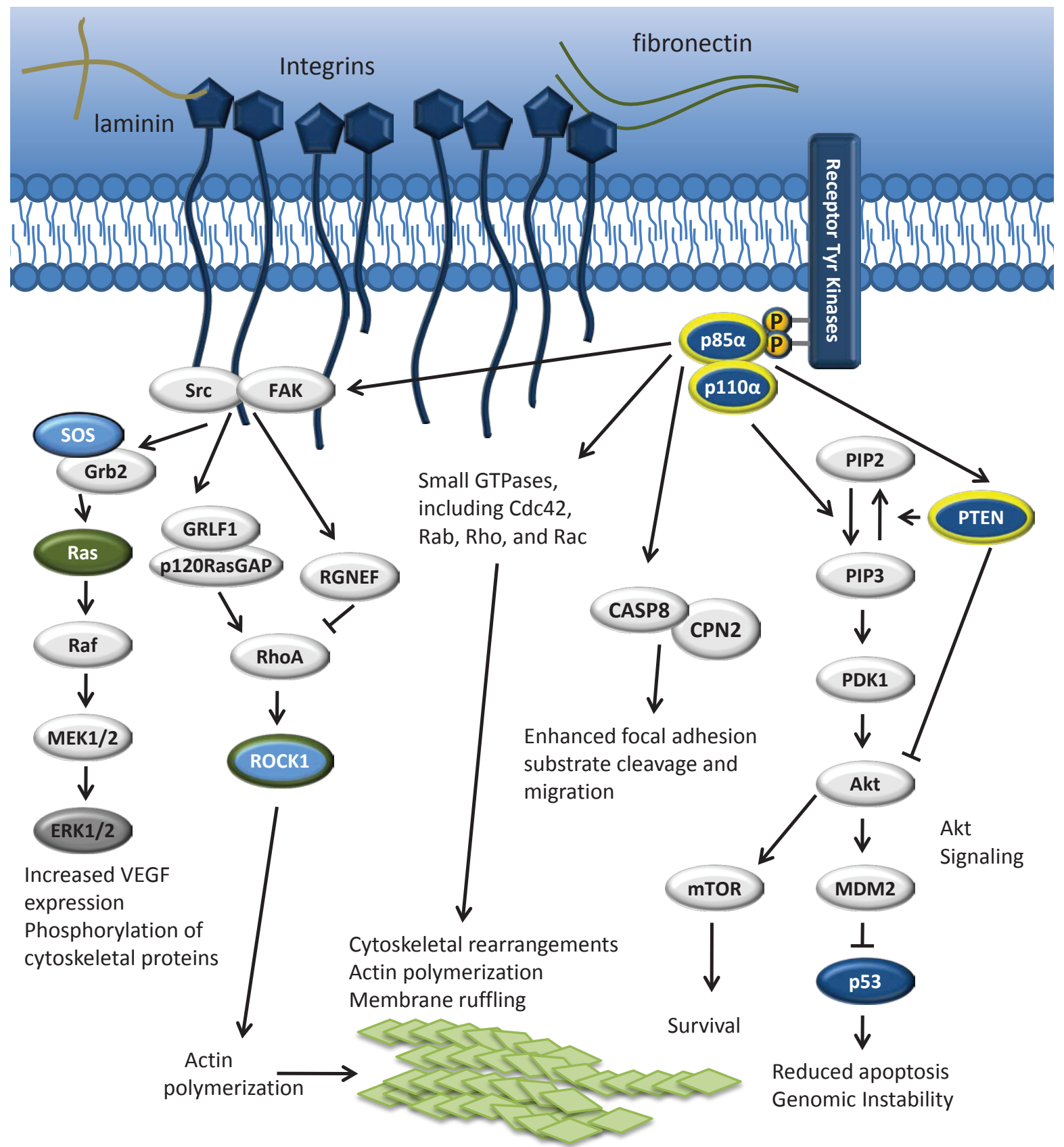

Figure Legend
PTEN Mutated from the Stringent Gene List
PEGR Mutated from the Full Gene List
POCK1 Both mutated in the full gene list and overexpressed
ERR1/2 Downregulated by at least 5-fold in tumors
HAS1 Neither mutated nor overexpressed (by at least 5-fold)

Cytoskeletal rearrangements

Stress fiber/focal adhesion modification

lamellipodia/fillopodia formation

Figure 1: The PI3K pathway is central to proliferation, migration, and invasion in GBM cells. Upon activation at the plasma membrane, the catalytic subunit of class IA PI3Ks phosphorylates phosphatidylinositol 4,5-bisphosphate (PIP2), converting it to phosphatidylinositol 3,4,5-triphosphate (PIP3). This results in the activation of several downstream pathways, including the Akt and mTOR pathways, which leads to increased proliferation and survival. PTEN activity converts PIP3 to PIP2. Interestingly, the p85 $\alpha$ regulatory subunit has been shown to bind PTEN directly and enhance its lipid phosphatase activity [62]. This subunit may also be involved in the activation of migration and invasion-promoting pathways, possibly through the activation of small GTPases such as Cdc42 and Rac [23, 63]. p85 $\alpha$ may interact with caspase 8 (in a non-apoptotic function of this caspase) to increase cell motility and Rac activation [55]. p85 $\alpha$ has also been shown to interact with FAK, and this interaction correlates with FAK activation [14]. 
cells, this constitutes a change from a hydrophilic to an intermediate side chain at amino acid 1047. In HCT116 cells, amino acid 1047 remains hydrophilic. We confirmed the presence of these mutations in both cell lines (data not shown). Previous work by Samuels et al found that knockdown of the wild-type PIK3CA allele in HCT116 lines increased proliferation and invasion in this cell line as compared to the parental line under the condition of serum starvation [22]. Similarly, removal of the mutant allele resulted in significantly decreased proliferation and invasion under the same conditions. These effects were apparent under normal serum conditions, but were not as significant. We obtained the mutant allele only (HPIK3CA MT \#125) and wild-type allele only (HPIK3CA WT \#123) lines from Dr. Bert Vogelstein (Johns Hopkins), to be applied as a comparison for our own studies.

Two glioblastoma stem-like neurosphere lines, without genome wide sequencing data, were produced in our laboratory from patient tumors, 081024 and 081110 . These and the D54 glioblastoma line were sequenced for PIK3CA mutations using published primer sequences (Table S1) [6]. While 081110 was devoid of mutations in this subunit, 081024 carried 3 mutations on exon 13, each of which has been documented as a SNP (NCBI dbSNP database) [43]. These mutations represented the following amino acid substitutions: V680L, E707K, and L719V. Two of these SNPs (V680L and L719V) were present in D54 cells. None of these substitutions changes the hydropathicity of the residue, and no other mutations were noted.

\section{$P I K 3 C A$ knockdown decreases Akt activation in GBM cell lines, and knockdown of either PIK3CA or PIK3R1 reduces FAK activity}

To study the effects of PIK3CA and PIK3RI abrogation in GBM, we significantly reduced protein levels of $\mathrm{p} 110 \alpha$ and $\mathrm{p} 85 \alpha$ using a lentiviral expression vector containing shRNAs targeting each mRNA independently. We knocked-down expression of each subunit separately (and in combination) in the SKMG26, D54, HCT116, 081024, and 081110 cell lines. We produced controls for each line containing only an empty lentiviral vector and no shRNA. Knockdown was confirmed by western blot (Fig. 2A). Although interaction between the PIK3R1 and PIK3CA gene products may be important in the stabilization of the catalytic subunit, we did not observe much decrease in $\mathrm{p} 110 \alpha$ protein levels upon knockdown of $\mathrm{p} 85 \alpha$.

A
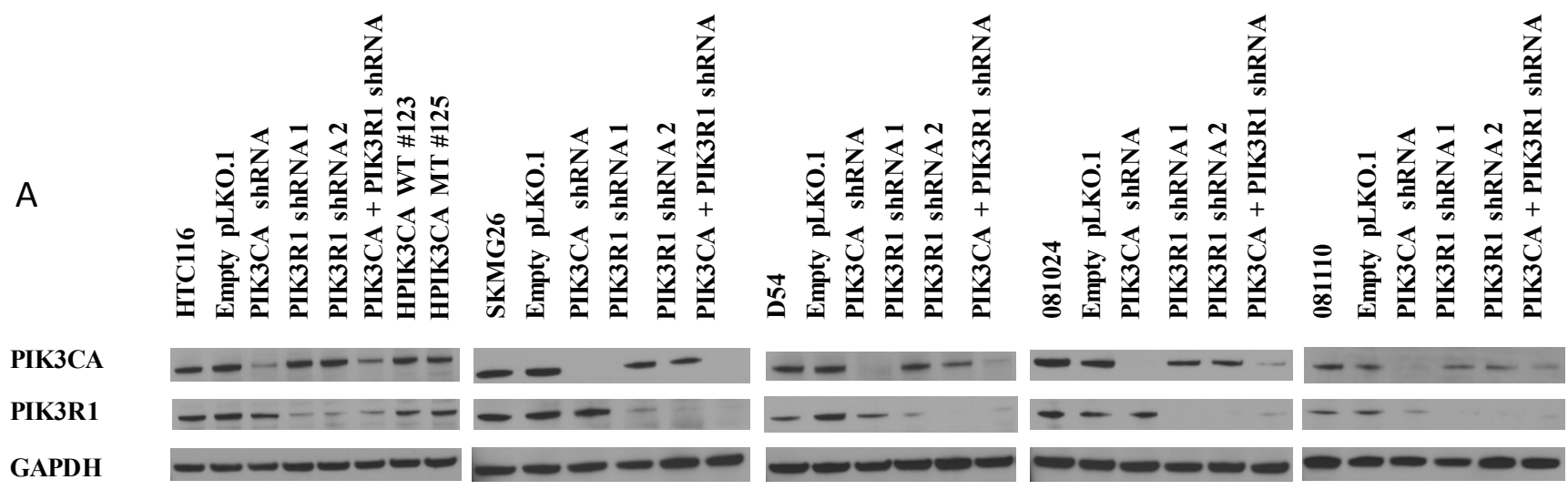

B
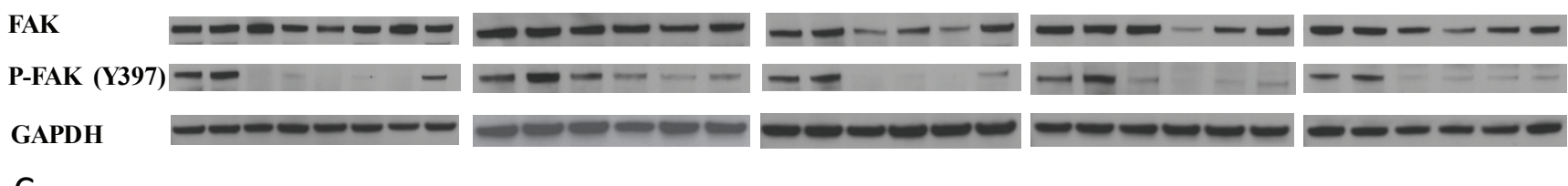

C
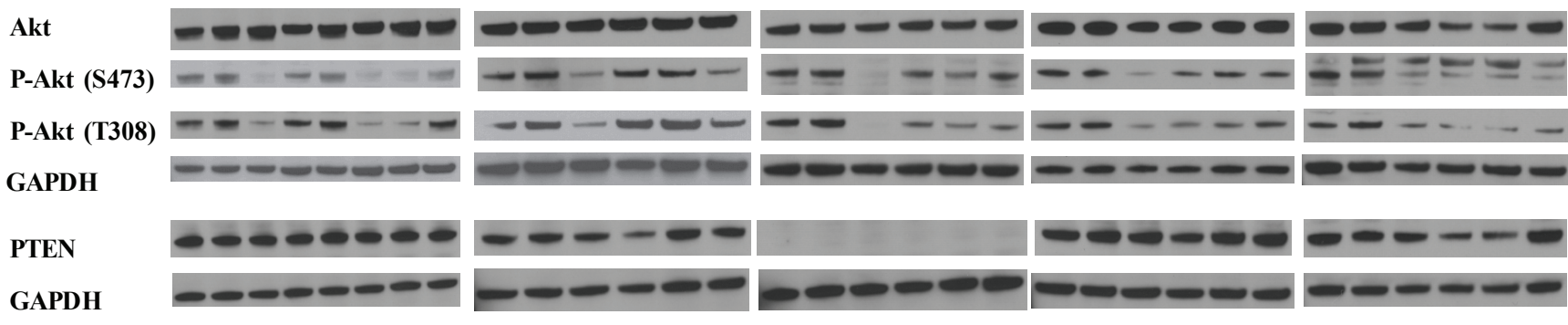

Figure 2: Confirmation of PIK3CA and PIK3R1 knockdown in cell lines. A, PIK3CA and PIK3R1 class IA PI3K subunit expression was reduced using lentiviral-mediated shRNA targeted to each subunit specifically, and to each in combination. B, FAK activation at Y397 was observed for each cell line. C, Akt activation at T308 and S473 was observed, as was PTEN status. 

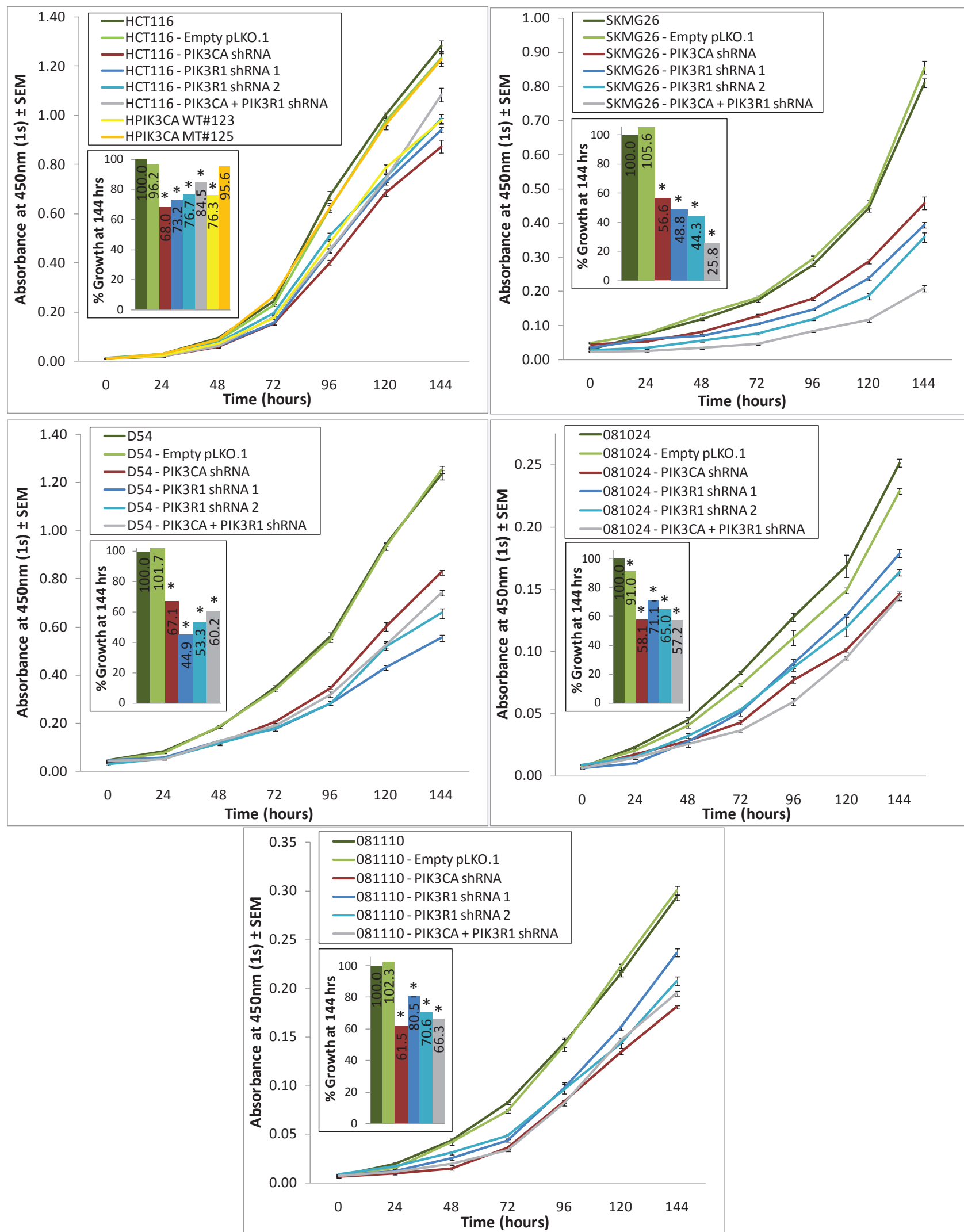

Figure 3: PIK3CA or PIK3R1 knockdown reduces proliferation of various cancer cell lines. Cells were plated at 500 or $1000 \mathrm{cells} / \mathrm{well}$ and proliferation was measured once per day using the Cell Counting Kit-8 reagent. At 144 hours, all PIK3CA and PIK3RI knockdown cell lines showed significantly reduced levels of growth as compared to parental and control lines. A more pronounced decrease in proliferation was noted in SKMG26 cells as a result of knockdown of both PIK3CA and PIK3R1. * represents a p-value $\leq 0.05$, and compares a given result to that of the parental line. 


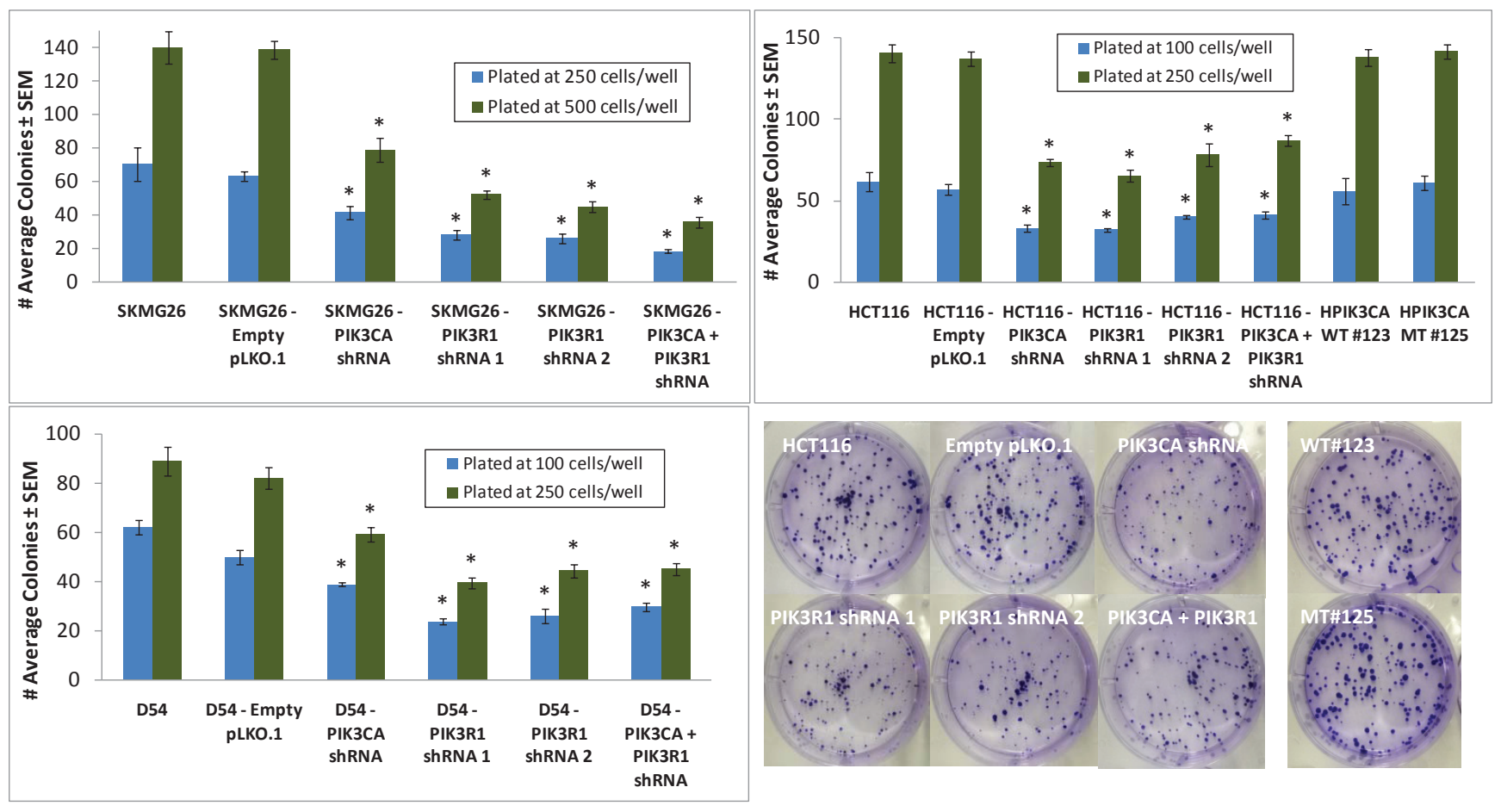

B

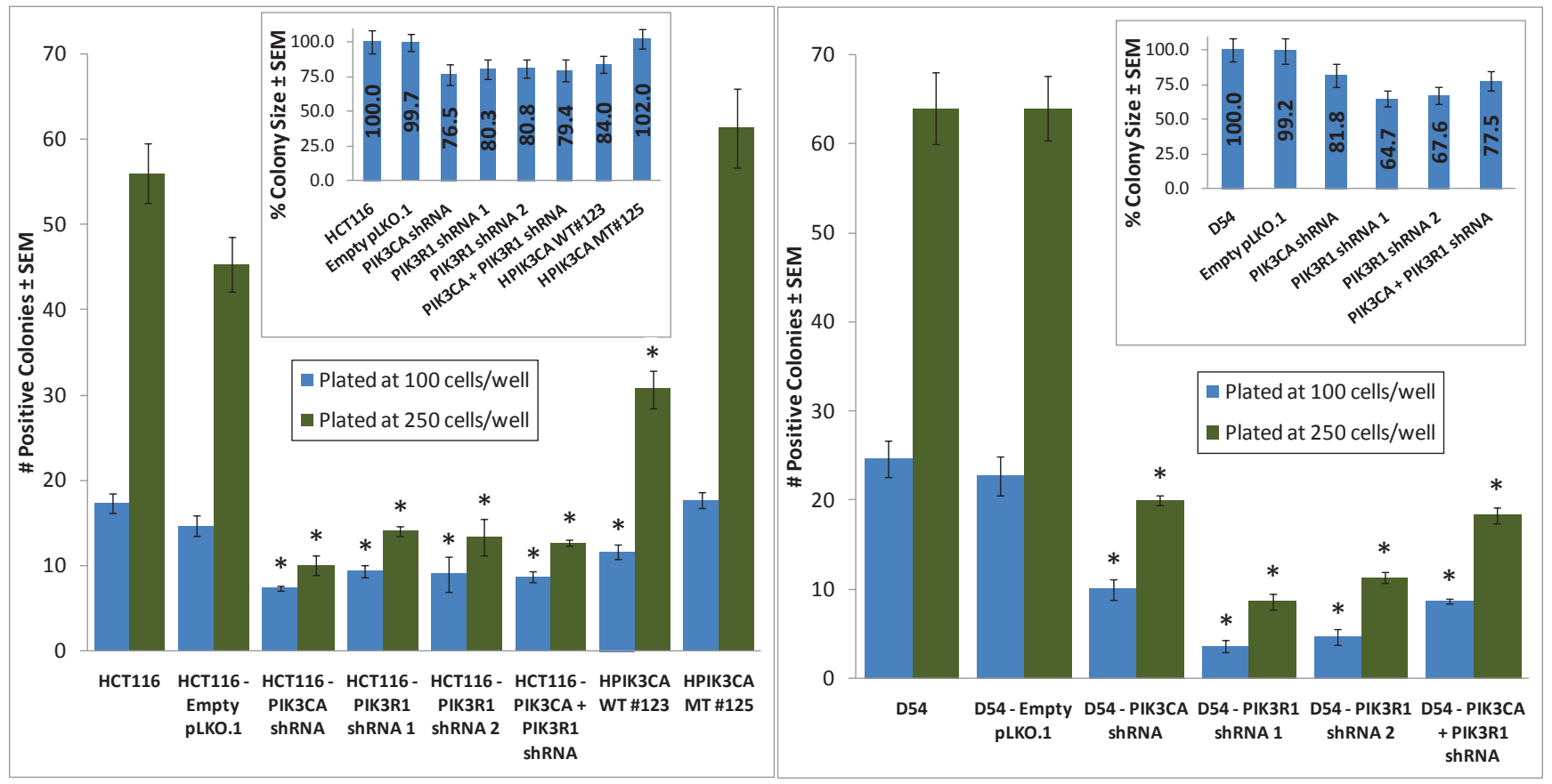

Figure 4: Colony formation is reduced on plastic and soft agar due to either PIK3CA or PIK3R1 knockdown. A, Cells were plated on treated plastic 6 -well plates at 100, 250, and 500 cells/well. Colonies were stained and counted at $\sim 2$ weeks post-plating. In all cells, PIK3CA or PIK3R1 knockdown resulted in a decrease in colony number. B, Cells were plated at 100 and 250 cells/well in top plugs containing appropriate media and $0.4 \%$ agarose. At $\sim 2$ weeks post-plating, colonies were stained, measured, and counted. A colony was counted as positive if it matched or exceeded the average size of colonies formed by the parental line. In all cell lines, $P I K 3 C A$ or $P I K 3 R 1$ knockdown reduced both colony size and number of positive colonies. * represents a p-value $\leq 0.05$, and compares a given result to that of the parental line. 
We examined the phosphorylation levels of Akt at Ser473 and Thr308 in HCT116, D54, and SKMG26 cell lines. Akt activation was significantly reduced at both residues in cells following PIK3CA knockdown (Fig.2C). In HCT116 lines, this is similar to the decrease in Akt activity seen in HPIK3CA WT \#123 lines, which contain only the wild-type PIK3CA allele. PIK3RI knockdown did not result in reduced Akt activation, and in the case of SKMG26, this seemed to increase levels of phosphorylation at both of the above residues. In the 081110 neurosphere line, Akt activity was slightly reduced by both $P I K 3 C A$ and $P I K 3 R 1$ knockdown. PIK3R1 and $P I K 3 C A$ knockdown in 081024 neurospheres resulted in a decreased level of Akt phosphorylation at both residues, which was more pronounced in the PIK3CA knockdown as compared to the PIK3R1 knockdown. Neither neurosphere line showed decreased Akt phosphorylation at levels as significant as in the three adherent lines. These results differentiates the 3 adherent lines from the 2 nonadherent neurosphere lines, suggesting that PIK3CA knockdown may not affect Akt activity as significantly in neurospheres as compared to adherent lines.

We also studied the activity of FAK (PTK2) in all lines at the Y397 residue (Fig.2B). Both PIK3R1 and
PIK3CA knockdown significantly decreased FAK activity in all lines tested.

\section{Knockdown of $P I K 3 C A$ or $P I K 3 R 1$ reduces proliferation in GBM cells in vitro, regardless of PIK3CA mutational status}

Abrogation of either the PIK3CA or PIK3R1 genes significantly reduced proliferation at 144 hours in all 5 cell lines (Fig. 3). Interestingly, in SKMG26 and D54 lines, PIK3R1 knockdown resulted in a more reduced proliferation rate than did $P I K 3 C A$ knockdown. The combined knockdown of PIK3CA and PIK3R1 only showed a greater effect than either knockdown alone in SKMG26 lines.Similarly, in SKMG26, D54, and HCT116 the colony formation capabilities of the PIK3CA and $P I K 3 R 1$ knockdown lines were reduced both on treated plastic and, more significantly, in soft agar (Fig. 4). In D54 and SKMG26 lines, both $P I K 3 C A$ and $P I K 3 R 1$ knockdown lines showed significantly reduced colony numbers on plastic as compared to parental lines and controls, with the greatest reduction due to $P I K 3 R 1$ abrogation $(\mathrm{p}<0.0001)$. In HCT116 cells, PIK3CA knockdown resulted in a

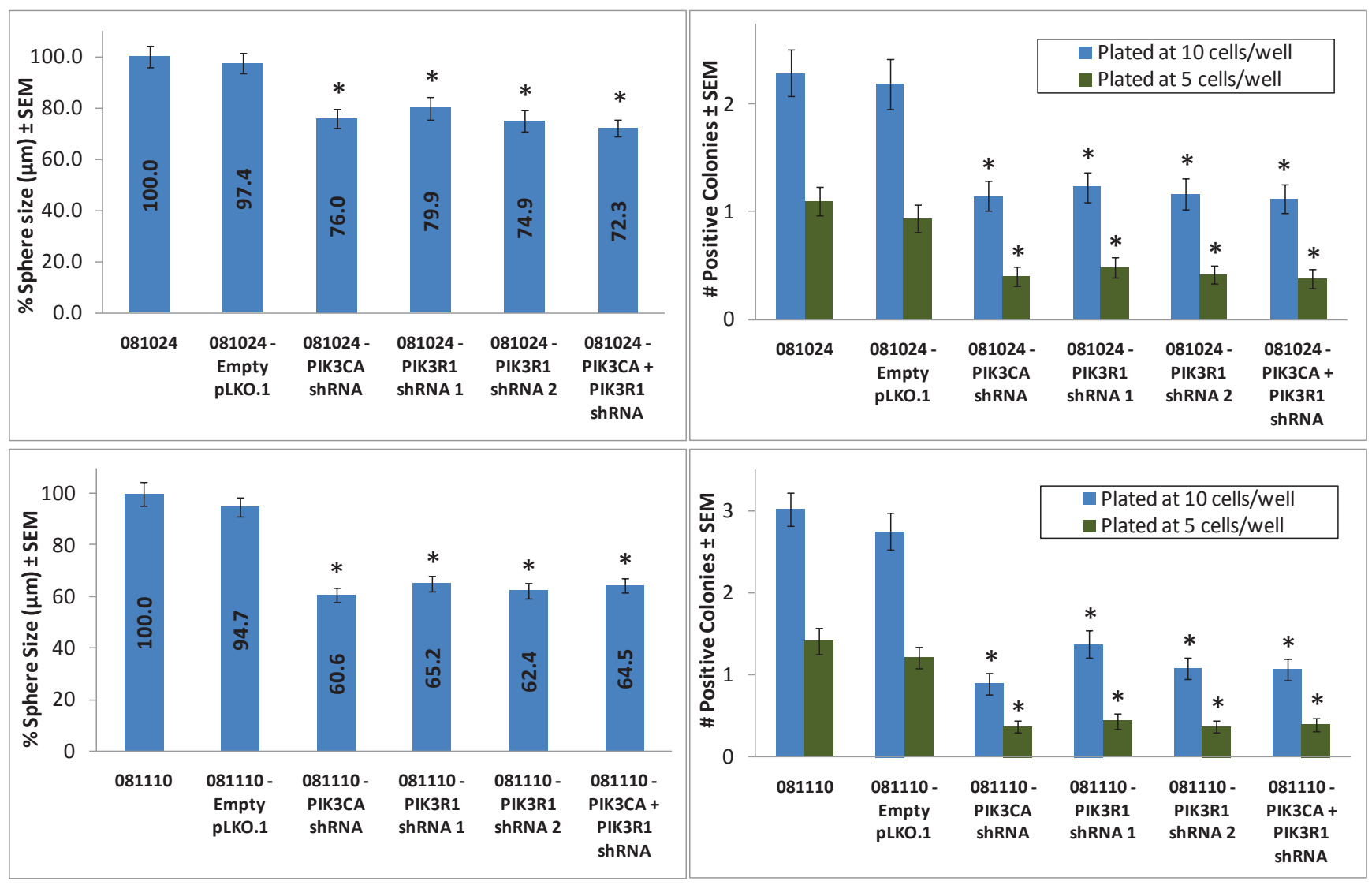

Figure 5: Neurosphere size and number is reduced in PIK3CA and PIK3R1 knockdown cell lines. Cells were plated at 1, 5, $10,100,250$, and 500 cells/well, and at 2 weeks post-plating, neurospheres were counted and measured. A sphere was considered positive if it matched or exceeded the average size of spheres formed by parental lines. In both 081110 and 081024 cell lines, PIK3CA and PIK3R1 knockdown resulted in reduced neurosphere size and number of positive spheres per well. * represents a p-value $\leq 0.05$, and compares a given result to that of the parental line. 
more reduced colony number than PIK3R1 knockdown, although this was not statistically significant.

In soft agar, a colony was counted as positive if it matched or exceeded the average size of colonies formed by the parental line. SKMG26 cells did not form colonies in soft agar at any agar concentration or cell number. In D54 cells, PIK3R1 knockdown resulted in a greater reduction in colony size and number of positive colonies than PIK3CA knockdown, while PIK3CA knockdown showed a slightly greater effect in HCT116 cells. HPIK3CA WT \#123 cells also exhibited reduced colony size and number, although this was not as significant as that seen in knockdown lines. HPIK3CA MT \#125 cells showed an increase in colony size and number, although

A
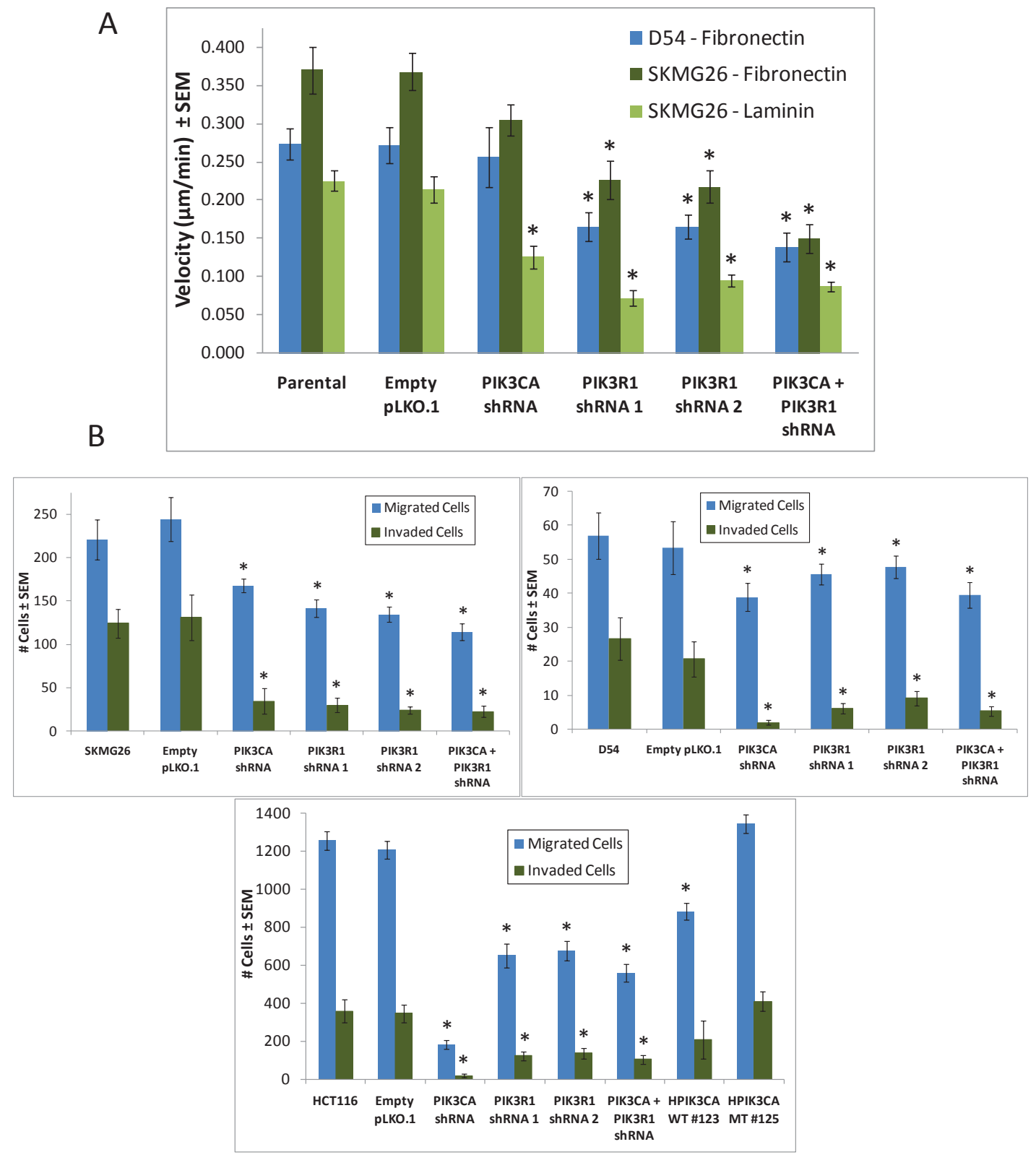

Figure 6: $P I K 3 C A$ knockdown results in reduced cell migration rates on fibronectin and laminin, and both $P I K 3 C A$ and PIK3R1 knockdown reduce invasion through Matrigel. A, Cells were plated on fibronectin or laminin and were imaged every 5 minutes for 24 hours. Average velocity and distance traveled were measured at 6, 8, 12, 16, and 24 hours, and trends were similar for each time point (graph represents the 16 hour time point). In both D54 and SKMG26 cells, PIK3CA knockdown did not result in a statistically significant reduced migration rate on fibronectin. $P I K 3 R 1$ knockdown reduced migration rates in both lines. D54 lines did not adhere to laminin-coated surfaces. In SKMG26 lines on laminin, both $P I K 3 C A$ and $P I K 3 R 1$ knockdown significantly reduced the average rate of cell migration, with the combined knockdown resulting in the greatest decrease in velocity. $*$ represents a $p$-value $\leq 0.05$, and compares a given result to that of the parental line. B, Cells were plated at $2.5 \times 10^{5}$ or $5 \times 10^{5}$ cells/well on BD Biocoat Matrigel Invasion Assay inserts. At 24 or 72 hours, migrated (control) and invaded (experimental) cells were stained and counted. Percent invasion is provided for each cell line. Both PIK3CA and PIK3RI knockdown resulted in a reduced percent invasion as compared to parental lines and controls. * represents a $\mathrm{p}$-value $\leq 0.05$, and compares a given result to that of the parental line. 
this was not statistically significant.

081024 and 081110 PIK3CA and PIK3RI knockdown lines formed significantly smaller colonies over 2 weeks than parental and control lines (Fig. 5). These data suggest that cells lacking $P I K 3 C A$ or $P I K 3 R 1$ experience significantly reduced survival under anchorage-independent conditions.

\section{Knockdown of PIK3CA or PIK3R1 reduces GBM cell invasion and migration on laminin and fibronectin}

Fibronectin and laminin are two components of brain extracellular matrix that have been found to be upregulated in glioblastoma, and both may play a role in tumor migration and invasion $[17,19]$. Evidence suggests that differential interaction with matrix components may affect the migrational and proliferative capabilities of tumor cell lines. Sengupta et al found that shRNAmediated inhibition of fibronectin expression delayed GBM tumor progression in mice [21]. Nagato et al showed that knockdown of laminin $\alpha 4$ chain severely limited tumor invasion in vivo [20].

Cells were plated on fibronectin or laminin and were imaged using an Olympus IX81 microsope for 24 hours in order to study the migrational capabilities of the knockdown cell lines. As HCT116 cell lines tend to divide quickly and grow in clumps, migration studies focused on the D54 and SKMG26 lines, which are more motile and therefore trackable. Cells were tracked individually using the Manual Tracking program in ImageJ [44, 45]. 081110 and 081024 lines were not tested in this capacity, as they did not adhere to laminin or fibronectin. On fibronectin, PIK3CA knockdown resulted in a reduced average velocity $(\mu \mathrm{m} / \mathrm{min})$ and distance traveled $(\mu \mathrm{m})$ in both SKMG26 and D54 cell lines as measured at $6,8,12$, 16 , and 24 hours, although not in a statistically significant manner (Fig. 6A, Videos S1-S3). PIK3R1 knockdown did significantly lower velocity and distance traveled in both cell lines (Videos S4-5). In SKMG26 cells (but not in D54 cells), combined PIK3CA and PIK3R1 knockdown resulted in the greatest decrease in overall velocity (Video S6). D54 cells did not adhere to laminin-coated surfaces. SKMG26 cells on laminin showed significantly reduced migration rates after knockdown of either the PIK3CA or PIK3R1 subunit (Fig. 6A). Migration velocity was decreased more drastically due to PIK3R1 than PIK3CA knockdown, and no combinatorial effect was observed due to combined knockdown. Overall, migration velocity was more greatly reduced on laminin as compared to cells observed on fibronectin. Cell migration rates were reduced in the $P I K 3 R 1$ knockdown line as compared to
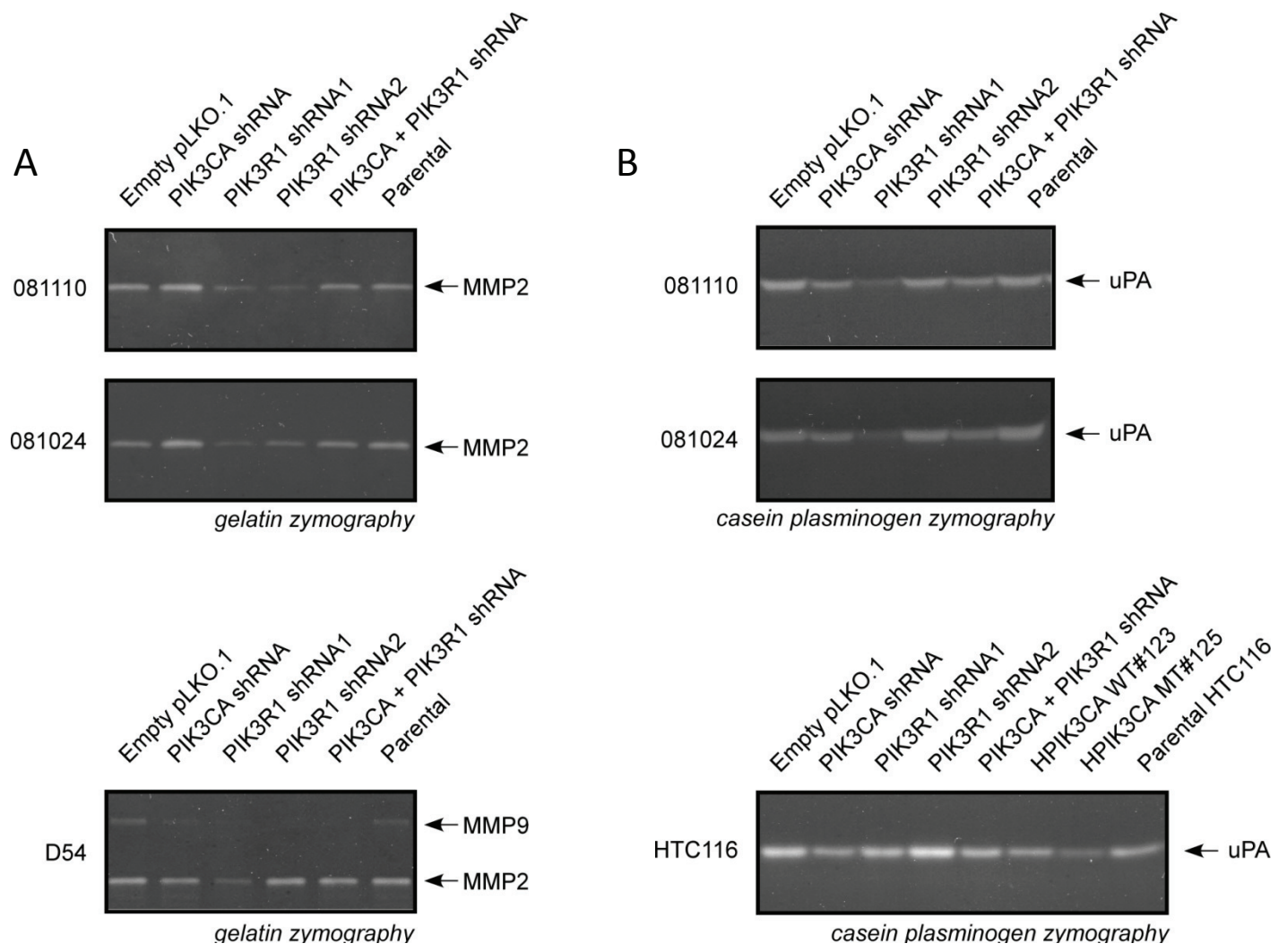

Figure 7: Protease activity is altered in PIK3R1 knock down cell lines. The 48 hour conditioned medium of adherent or suspension cell lines was collected and subjected to gelatin or casein-plasminogen zymography. PIK3R1 knock down resulted in reduced MMP-2 and uPA activity in neurosphere lines. MMP-9 was reduced in all knock downs in D54 cells. Knock down or mutation of $P I K 3 C A$ resulted in decreased uPA in HTC116 cells. 
the parental SKMG26 line by $68.0 \%$ on laminin versus $38.8 \%$ on fibronectin at 16 hours.

We used the BD Biocoat Matrigel Invasion Assay to test the invasive capabilities of SKMG26, D54, and HCT 116 cells lacking $P I K 3 C A$ or $P I K 3 R 1$ mRNA. For all 3 lines, both $P I K 3 R 1$ and $P I K 3 C A$ knockdown significantly reduced the number of invaded cells versus controls, with the lowest percent invasion in PIK3CA knockdown lines (Fig. 6B). Together, these data suggest that PIK3RI plays an important role in the rate of migration, while $P I K 3 C A$ loss may lead to a greater reduction in invasive capabilities. Both of these characteristics are likely to be important in the infiltration of healthy non-tumor brain tissue by GBM cells.

\section{MMPs and uPA are reduced as a result of knockdown}

Gelatin and casein plasminogen zymograms showed that treatment with PIK3R1 shRNA resulted in a decrease in MMP2 and uPA activity in both 081024 and 081110 neurosphere lines (Figure 7). PIK3CA knockdown caused no change in activity. In D54 lines, MMP2 activity was similarly reduced by $P I K 3 R 1$ knockdown, but not by PIK3CA knockdown. D54 cells also express MMP9, which was reduced in all knockdowns as compared to control lines.

\section{DISCUSSION}

Invasion and migration-related pathways were among the most statistically significantly enriched for mutations and expression alterations in our informatics studies using Ingenuity, Partek, and Spotfire. Central to many of these pathways is PI3-Kinase signaling. The validity of our results is strengthened by the similarly significant presence of pathways involved in cell cycle control, apoptosis, and proliferation (Tables S4-S7).

Much research has focused on elucidating the mechanisms of tumor formation and progression caused by individual common mutations, such as PTEN and p53 loss of function, EGFR amplification, or the EGFRvIII variant. However, considering the pathways that are enriched for aberrations may give a more comprehensive view of tumor function. Although we have not addressed epigenetic mechanisms in this study, these should not be excluded from further considerations of the underlying mechanisms for promoting invasion.

Our informatics studies focused primarily on pathways that may affect motility in GBM cells. We found that focal adhesion signaling, integrin signaling, and extracellular component and receptor signaling commonly contained clusters of mutations and/or aberrantly expressed genes (Fig. S1-S3). These signaling pathways are highly interconnected, and most certainly experience altered communication in tumors. While many of the mutations found may be passengers, and thus may not support tumor growth or motility, some, such as mutations found in receptor tyrosine kinases, have already been shown to play a role in cancer [46].

Several integrins are upregulated in GBM, and knockdown of these proteins results in reduced migration and invasion [47, 48]. Our studies confirmed the upregulation of a collection of integrin subunits as compared to normal brain samples by over 5 -fold, including $\alpha \mathrm{v}$ (8.6-fold), $\alpha 2$ (8.6-fold), $\alpha 4$ (36.9-fold), and $\beta 8$ (8.3-fold). Several other subunits were upregulated at lower levels, including $\beta 3$ (3.6-fold). Each of these subunits was expressed in at least half of the tumors studied. The $\alpha 4$ subunit was also found to bear a mutation. Matsunaga et al. showed that knockdown of VLA-4 in acute myelogenous leukemia cells resulted in increased susceptibility of to anoikis- or drug-induced apoptosis [49]. This was likely caused by a reduction in PI3K activity, which is activated by the interaction of the $\alpha 4$ integrin subunit of VLA-4 and fibronectin. Treatment of mice by the same group with an anti-VLA-4 antibody resulted in 100\% survival at 62 days.

At least partially dependent on integrin signaling, focal adhesion turnover is likely to be highly important in the migration of tumor cells through healthy brain tissue $[12,14]$. The FAK protein itself was neither mutated nor dysregulated according to our studies, although it has been shown to be upregulated in a subset of breast tumors [50]. However, several proteins that may influence the activity of this pathway were upregulated and/or mutated in GBM by our findings. This includes the integrins discussed above, several of which, such as the $\beta 3$ subunit, activate FAK signaling. Similarly, activated EGFR and PDGFRA, both of which are upregulated (61.7- and 6.8-fold, respectively) and mutated in GBM, can aid in the activation of the FAK pathway [51]. PI3K, PTEN, RAP1B, TLN1, SOS, MAP3K11, TTN, BCAR3, and ROCK1 were each mutated in GBM in our studies, and ROCK1 was found to be upregulated by 8.4 -fold as compared to normal brain. NRAS, ARPC1B (subunit 1B of the Arp2/3 complex), PDGFC, PDGFD were upregulated by 13.7-, 5.4-, 16.3, and 5.5-fold respectively. Interestingly, Fotiadou et al found that mouse embryonic fibroblasts lacking NRAS also lacked phosphorylated FAK, and knockdown of NRAS may be useful in reducing migration in tumors[52].

Extracellular matrix components and their cell receptors play an important role in both integrin signaling and FAK signaling as discussed above. Many structural matrix components are overexpressed or mutated in GBM, and tumor cells may in fact express components that can increase their migratory capabilities $[17,19]$. The fibronectin 1 (FN1) gene carried a mutation in 1 tumor, and the $C O L 3 A 1$ gene was considered a potential driver by Parsons et al., with 3 mutations in individual tumors [6]. Several other collagen genes were mutated (COL14A1, 
COL23A1, COL4A2, COL4A2, COL4A5, COL6A3, and COL6A6, and COL3A2 was found to amplified by Rao et al [33]. FN1, 2 laminin genes (LAMC1, LAMA4), and a collection of collagen genes (COL1A1, COL20A1, COL5A1, COL4A2, COL1A2, COL12A1, COL22A1, COL6A1, COL28A1, COL6A3) were upregulated in tumors as compared to normal brain. Several additional collagen genes were overexpressed, but were not expressed in at least half of the tumors studied.

Given that rapid cell proliferation is inherent in GBM and is responsible for the production of the main tumor mass, the roles played by PI3K signaling in proliferation and tumor growth are most commonly studied in GBM. However, our results show that the PIK3CA gene, encoding the class IA PI3K catalytic subunit $100 \alpha$, and the PIK3R1 gene, encoding the $\mathrm{p} 85 \alpha$, p $55 \alpha$, and $\mathrm{p} 50 \alpha$ regulatory subunits, may also be key components of the migratory/invasive phenotype in these tumors. Interestingly, while knockdown of either of these subunits reduced proliferation, migration, and invasion in all cell lines tested, the extent of these effects differed slightly in each line. A measurably greater effect resulting from combined PIK3CA and PIK3R1 knockdown, as compared to knockdown of a single gene alone, was observed only in SKMG26 cells. Our studies show that the effects caused by abrogation of PIK3R1 and PIK3CA result from different signaling mechanisms in individual cell lines, likely reflecting different signaling mechanisms in the individual tumors (and perhaps in individual cells of the same tumor). . Although signaling responses to $P I K 3 C A$ and PIK3R1 knockdown differ among cell lines, proliferation, migration, and invasion were decreased in all lines tested.

p $85 \alpha$ activity in the invasive/migratory phenotype has yet to be fully elucidated. p85 $\alpha$ may, at least in part, act through focal adhesion signaling, as knockdown results in decreased FAK activation. Cells treated with $P I K 3 R$ l-targeting shRNA show a more reduced average velocity than cells treated with $P I K 3 C A$-targeting shRNA. However, these cells are still motile on both fibronectin and laminin, and thus the FAK signaling pathway is likely only one component of GBM cell motility. Notably, while both $P I K 3 C A$ and $P I K 3 R 1$ knockdown significantly reduces FAK activity in D54 glioblastoma cells, only reduction in PIK3R1 expression results in statistically significantly slowed cell migration, supporting the only partial role played by FAK signaling in GBM cell motility. The $\mathrm{p} 85 \alpha$ protein product of $P I K 3 R 1$ has been shown to interact with several small GTPases, including Cdc42, Rac, and Rho, that through various mechanisms increase cell motility [23, 53]. Park et al found that mir-29 microRNAs upregulate p53 levels, and induce apoptosis, potentially by direct suppression of PIK3RI and CDC42 [54]. p85 $\alpha$ may also interact with caspase- 8 to stimulate motility through Rac activation [55]. Similarly, PIK3R1 knockdown reduced MMP2 and uPA levels in neurospheres, and reduced MMP2 and MMP9 levels in D54 cells, and reduction of the levels of these proteins has been shown to cause reduced migratory and invasive capacity in glioblastoma cells $[56,57]$.

There are a variety of small molecule inhibitors available that target $\mathrm{p} 110 \alpha$, albeit non-specifically, including LY294002 and wortmannin. These and other PI3K inhibitors have been shown to reduce angiogenesis, induce radiosensitivity, and reduce proliferation in GBM cells [58-61]. Although PIK3R1 has not been studied previously in the context of GBM invasion, given its role in the PI3K pathway, and the 2008 discovery of its mutation in GBM, it is not surprising that PIK3RI knockdown shows evidence of decreased invasion in vitro [6]. However, there is currently no inhibitor targeting $\mathrm{p} 85 \mathrm{\alpha}$. Based on the concept that a reduction in migration may sensitize tumor cells to apoptosis, the development of $\mathrm{p} 85 \alpha$ inhibitors used in combination with apoptosisinducing chemotherapies may be effective in treatment of patients with GBM.

Although much of what has been shown in this study has been implicated previously in other cancers, our findings support the hypotheses that the PI3K signaling pathway has the most significantly mutated genes driving cellular invasion. Within the common mutations that promote GBM formation and growth, those within members of this pathway (PTEN, p110 $\alpha, \mathrm{p} 85 \alpha$ and likely receptor tyrosine kinases such as EGFR) are initiating points for signaling enhanced invasion.. Considering the frequency of mutations in the PI3K pathway and its role in growth and invasion, it would be reasonable to increase efforts to develop molecular therapies targeting its pathological signaling in GBM.

\section{MATERIALS AND METHODS}

\section{Data Collection and Generation of Gene Lists}

This study focused on the results of a wholegenome sequencing analysis of Glioblastoma tumors by Parsons et al [6]. We analyzed genes found to be mutated via sequencing, along with 'target' genes identified as amplified or deleted by Illumina SNP arrays. We included mutated GBM genes found to be likely drivers of tumor formation and progression by Carter et al [7]. This analysis also included genes amplified or deleted in more than $2 \%$ of cases based on a combination of digital karyotyping and a review of published studies by Rao et al [33]. In total, 703 genes were found to be altered in GBM via these data sources.

Since it was likely that many of the low frequency mutations were passengers, a more stringent gene list was also created that included mutations with higher probabilities of being drivers in the formation and invasion 
of GBM. This list included 'target' genes identified as amplified or deleted by Parsons et al [6], CAN genes from the same group that had a passenger probability (low) $\mathrm{p}$-value of 0.05 or better, genes found by Rao et al [33] with a $4 \%$ or greater occurrence, and genes determined by Carter et al [7] to be likely drivers via their CHASM program. This stringent list included a total of 59 genes and was analyzed separately from the full list of 703 genes.

\section{Informatics Analysis of Mutated Gene Lists}

Our objective in this analysis was to identify genes, functions, or pathways that are involved in the invasive tumor phenotype and are enriched in GBM. To identify these invasion-related components, we analyzed our lists of GBM-specific mutated, deleted, and/or amplified genes using the Ingenuity Program (Ingenuity Systems) and the Partek Genomics Suite Program (Partek Inc.). The Partek Genomics Suite Gene Ontology function allows for the analysis of gene ontologies (based upon molecular function, cellular component, and biological process as defined by the Gene Ontology Consortium) enriched in GBM tumors. Based on a gene list, Partek assigns statistical significance to each function, component, and process ontology enriched within the list. Statistical significance in this program is defined by an 'Enrichment Score,' which is the negative $\log _{e}$ transformation of the p-value for the particular ontology. Enrichment Scores above $3(\mathrm{p} \leq 0.05)$ are considered statistically significant. The Ingenuity program analyzes genes based upon gene function (as opposed to inclusion in an ontology) and organizes results by p-value into statistically significant canonical pathways and networks. We analyzed the original gene list (703 genes) and the stringent gene list (59 genes) separately using both of these programs.

Additionally, within the identified statistically significant mutation-enriched pathways, the Spotfire DecisionSite program (TIBCO Software Inc.) was utilized to identify up- or down-regulated genes based on expression data from Parsons et al [6]. This gene expression data included 20,619 curated genes in 16 human glioblastoma tumors.

\section{Cell lines}

081110 and 081024 non-adherent neurosphere lines were produced in our laboratory from human tumor samples received immediately following resection from consented GBM patients. Neurospheres were cultured in NeuroCult NS-A basal medium (StemCell Technologies, Vancouver, BC, Canada) supplemented with $10 \mathrm{ng} / \mathrm{mLFGF}$ (PeproTech, Rocky Hill, NJ), 10ng/mL EGF (PeproTech), and $0.0004 \%$ heparin (StemCell Technologies). HCT116 cells and HCT116 lines containing only the wild-type (HPIK3CA WT \#123) or mutant (HPIK3CA MT \#125)
PIK3CA alleles were obtained from Dr. Bert Vogelstein (Johns Hopkins), and were grown in McCoy's Modified 5A medium (Sigma-Aldrich) supplemented with $10 \%$ FBS. D54 and SKMG26 cells were grown in DMEM (Sigma-Aldrich) supplemented with 10\% FBS.

\section{Lentivirus production}

shRNAs targeting the PIK3CA and PIK3R1 mRNAs cloned into the vector pLKO.1 and packaged into E.coli were obtained from the Expression Arrest-TRC shRNA Libraries (Broad Institute of MIT and Harvard). The plasmid was amplified on ampicillin-treated plates and colonies were then incubated in $6 \mathrm{~mL}$ LB Broth (Invitrogen) overnight at $37^{\circ} \mathrm{C}$ and $300 \mathrm{RPM}$. The plasmid was purified using the QIAprep Spin Miniprep Kit (Qiagen) as described by the manufacturer. 293T cells in $10 \mathrm{~cm}$ dishes were treated with Lipofectamine 2000 (Invitrogen), envelope plasmid pMD2.G, packaging plasmid pCMVR8.74psPAX2, and hairpin-pLKO.1 plasmid. At 18 hours post treatment, the cells were washed and fresh highBSA media was added. Lentivirus-containing media was collected at 24 and 48 hours and was stored at $-80^{\circ} \mathrm{C}$ until used. Lentivirus was also produced that contained only the empty pLKO.1 plasmid (no shRNA).

HCT116, D54, SKMG26, 081110, and 081024 cell lines were treated with lentivirus targeting either PIK3CA or PIK3R1 plus $8 \mathrm{ug} / \mathrm{mL}$ polybrene (Sigma-Aldrich). Lines were treated in parallel with a combination of both PIK3CA and PIK3R1 lentiviruses, and with lentivirus containing only the empty pLKO.1 plasmid (no shRNA) as a control. At 24 hours, cells were washed, and shRNAexpressing cells were selected with a concentration of puromycin (Invitrogen) ranging from $0.5-2 \mathrm{ug} / \mathrm{mL}$ for 72 hours. Cells were then maintained without selection.

\section{Sequencing}

Sequencing was performed as previously described [29]. Briefly, genomic DNA was purified from cell pellets using the DNeasy Blood and Tissue kit (Qiagen). Exons $1,2,4,5,7,9,12,13,18$, and 20 were amplified using PCR (primers listed in Table S1). PCR products were run on $2 \%$ agarose gels, and bands were extracted using the QIAQuick Gel Extraction Kit (Qiagen). Samples were sequenced by GeneWiz (South Plainfield, NJ).

\section{Growth curves}

Cells were treated with $2 \mathrm{ug} / \mathrm{mL}$ propidium iodide (Sigma-Aldrich) and were sorted by a MoFlo cell sorter (Dako Cytomation) into 96-well plates at 500, 1000 and 2000 cells/well. For 7 days post plating, live cells were counted daily in 10 replicates per line per day using the Cell 
Counting Kit-8 reagent (Enzo Life Sciences). Absorbance was read at $450 \mathrm{~nm}$ for 1 second. Results were normalized to wells containing media only (no cells).

\section{Colony formation assay}

Cell were plated in triplicate at 100,250 , and 500 cells/well in 6-well plates in appropriate medium with $10 \%$ FBS. At two weeks post-plating, colonies were stained with crystal violet (Sigma-Aldrich) and counted under a light microscope.

\section{Colony formation in soft agar}

Cells were plated in triplicate at 100 and 250 cells/ well in top plugs containing appropriate media plus $0.4 \%$ SeaPlaque agarose (Lonza). Bottom plugs contained media plus $0.8 \%$ agarose. At approximately two weeks post-plating, colonies were counted and measured under a light microscope.

\section{Neurosphere formation assay}

Cells were treated with $2 \mathrm{ug} / \mathrm{mL}$ propidium iodide and live cells only were sorted using a MoFlo cell sorter (Cytomation) into 96-well plates at 1, 5, 10, 100, 250, and 500 cells/well. At two weeks post plating, neurospheres were measured for average size and the number of positive neurospheres was recorded at each cell concentration. Neurospheres were considered positive if they matched or exceeded a measurement close to the average size of neurospheres formed by the parental cell line.

\section{In vitro invasion assay}

The BD Biocoat Matrigel Invasion Assay was performed as described by the manufacturer (Becton Dickson). Matrigel inserts were rehydrated with appropriate medium and cells were plated at $2.5 \times 10^{5}$ or $5 \times 10^{5}$ cells/well in triplicate in both control and experimental inserts. At 24 or 72 hours, cells remaining in the upper chamber were scraped and invaded cells were stained with crystal violet. Cells were counted using light microscopy on a $1 \mathrm{~mm}^{2}$ grid. Two $1 \mathrm{~mm}^{2}$ sections were counted at the edge and two at the center of each insert.

\section{Migration assays on laminin and fibronectin}

Cells were plated in triplicate at 10,000 cells/well in 48-well plates coated with laminin or fibronectin (Becton Dickson). At 48-hours post-plating, cells were imaged every 5 minutes for 24 hours at two points per well on an Olympus IX81 microscope (Olympus) with SlideBook
4.2 software (Intelligent Imaging Innovations, Inc.). Cells were maintained in a humidity chamber during imaging at $37^{\circ} \mathrm{C}$ and $5 \% \mathrm{CO}_{2}$. Cells were tracked using the Manual Tracking plug-in in ImageJ [44, 45]. Average distance travelled and average velocity were measured for each line at $6,8,12,16$, and 24 hours.

\section{Gelatin zymography and casein plasminogen zymography}

Equal amount of protein from cell-conditioned medium was loaded onto a $10 \%$ polyacrylamide gel (Sigma-Aldrich) with either $1 \mathrm{mg} / \mathrm{mL}$ gelatin or $1.5 \mathrm{mg} / \mathrm{mL}$ casein and 0.28 units $/ \mathrm{mL}$ plasminogen for zymography. After SDS-PAGE separation, gels were incubated in renaturing buffer (Invitrogen) to remove SDS and help the renaturation of proteases. The gels were then incubated in developing buffer (Invitrogen) at $37^{\circ} \mathrm{C}$ for 18 hours. Gels were stained with $0.25 \%$ Coomassie Blue R-250 (Thermo Scientific) in 45\% methanol, 10\% acetic acid, and destained in methanol:water:acetic acid (2.5:6.5:1). No digestion was detected in control casein gels where plasminogen was omitted, confirming the plasminogen activator nature of the uPA band.

\section{Immunoblotting}

Protein levels were measured by immunoblotting using antibodies specific to p110 $\alpha$, p $85 \alpha$, FAK, PhosphoFAK (Y397), Akt, Phospho-Akt (T308), Phospho-Akt (S473) (Cell Signaling Technology, Danvers, MA), PTEN (Abcam, Cambridge, MA), and GAPDH (Santa Cruz Biotechnology). Whole cell lysates transferred onto PVDF membranes were blocked with 5\% BSA prior to treatment with antibodies. Following secondary antibody treatment and thorough washing, membranes were treated with the SuperSignal West Pico Chemiluminescent Substrate (Thermo Scientific). Membranes were developed in a dark room using autoradiography film.

\section{Statistical Analyses}

Data were analyzed using the student's t-test. A result with a p-value of less than or equal to 0.05 was considered significant.

\section{ACKNOWLEDGEMENTS}

We would like to thank Conover Talbot for his help with our understanding of the Partek, Ingenuity, and Spotfire software, Dr. Hao Zhang for the sorting of our cell lines at the Bloomberg School of Public Health Cell Sorting Facility, and Dr. Avadhut Joshi for his continually helpful discussions. This project was supported by NIH 
Grant R01 NS052507 and the Virginia and D.K. Ludwig Fund for Cancer Research. ZAB was supported by NIH grant T32CA130840, the Postdoctoral Training Program in Nanotechnology for Cancer Medicine. GJR is supported by the Irving J. Sherman M.D. Neurosurgery Research Professorship.

\section{SUPPLEMENTARY VIDEOS}

Videos S1-S6. Videos of SKMG26 (Video S1), SKMG26 - Empty pLKO.1 (Video S2), SKMG26 - PIK3CA shRNA (Video S3), SKMG26 - PIK3R1 shRNA 1 (Video S4), SKMG26 - PIK3R1 shRNA 2 (Video S5), and SKMG26 - PIK3CA + PIK3R1 shRNA (Video S6) cell lines tracked on fibronectin using the Manual Tracking plug-in in ImageJ. Individual tracks are provided for all trackable cells in each video. The videos, supplementary figures and tables can be found online at www.impactjournals.com/oncotarget

\section{REFERENCES}

1. Stupp R, Mason WP, van den Bent MJ, Weller M, Fisher B, Taphoorn MJB, Belanger K, Brandes AA, Marosi C, Bogdahn U. Radiotherapy plus concomitant and adjuvant temozolomide for glioblastoma. New England Journal of Medicine. 2005; 352:987-996.

2. Burger PC, Dubois PJ, Schold Jr SC, Smith Jr KR, Odom GL, Crafts DC, Giangaspero F. Computerized tomographic and pathologic studies of the untreated, quiescent, and recurrent glioblastoma multiforme. Journal of Neurosurgery. 1983; 58:159-169.

3. Silbergeld DL, Chicoine MR. Isolation and characterization of human malignant glioma cells from histologically normal brain. Journal of Neurosurgery. 1997; 86:525-531.

4. Rao JS. Molecular mechanisms of glioma invasiveness: the role of proteases. Nature Reviews Cancer. 2003; 3:489501.

5. Lefranc F, Brotchi J, Kiss R. Possible future issues in the treatment of glioblastomas: special emphasis on cell migration and the resistance of migrating glioblastoma cells to apoptosis. Journal of clinical oncology. 2005; 23:2411.

6. Parsons DW, Jones S, Zhang X, Lin JC, Leary RJ, Angenendt P, Mankoo P, Carter H, Siu IM, Gallia GL, Olivi A, McLendon R, Rasheed BA, Keir S, Nikolskaya T, Nikolsky $Y$ et al. An Integrated Genomic Analysis of Human Glioblastoma Multiforme. Science. 2008; 321:1807-1812.

7. Carter H, Chen S, Isik L, Tyekucheva S, Velculescu VE, Kinzler KW, Vogelstein B, R K. Cancer-Specific High-Throughput Annotation of Somatic Mutations: Computational Prediction of Driver Missense Mutations. Cancer Research. 2009; 69:6660-6667.

8. Giese A, Loo MA, Tran N, Haskett D, Coons SW, Berens ME. Dichotomy of astrocytoma migration and proliferation.
International journal of cancer. 1996; 67:275-282.

9. Giese A, Bjerkvig R, Berens M, Westphal M. Cost of migration: invasion of malignant gliomas and implications for treatment. Journal of clinical oncology. 2003; 21:1624.

10. Goswami S WW, Wyckoff JB, Condeelis JS. Breast cancer cells isolated by chemotaxis from primary tumors show increased survival and resistance to chemotherapy. Cancer Res. 2004; 64:7664-7667.

11. Giese A RM, Loo MA, Berens ME. Determinants of human astrocytoma migration. Cancer Res. 1994; 54:3897-3904.

12. Sieg DJ, Hauck CR, Ilic D, Klingbeil CK, Schaefer E, Damsky CH, Schlaepfer DD. FAK integrates growth-factor and integrin signals to promote cell migration. Nature cell biology. 2000; 2:249-256.

13. Guo W, Giancotti FG. Integrin signalling during tumour progression. Nature reviews Molecular cell biology. 2004; $5: 816$.

14. Zeng ZZ JY, Hahn NJ, Markwart SM, Rockwood KF, Livant DL. Role of focal adhesion kinase and phosphatidylinositol 3'-kinase in integrin fibronectin receptor-mediated, matrix metalloproteinase-1-dependent invasion by metastatic prostate cancer cells. Cancer Res. 2006; 66:8091-8099.

15. Michael KE, Dumbauld DW, Burns KL, Hanks SK, Garcia AJ. Focal adhesion kinase modulates cell adhesion strengthening via integrin activation. Molecular biology of the cell. 2009; 20:2508.

16. Liu D, Martin V, Fueyo J, Lee OH, Xu J, Cortes-Santiago N, Alonso MM, Aldape K, Colman H, Gomez-Manzano C. Tie2/TEK Modulates the Interaction of Glioma and Brain Tumor Stem Cells with Endothelial Cells and Promotes an Invasive Phenotype. Oncotarget. 2010; 1:700-709.

17. Sönmez H KE, Süer S, Ozyurt E. Fibronectin and sialic acid levels in human meningiomas and gliomas. Cancer Letters. 1995; 90:119-122.

18. Ohnishi T HS, Izumoto S, Matsumura H, Kanemura Y, Arita $\mathrm{N}$, Hayakawa T. Role of fibronectin-stimulated tumor cell migration in glioma invasion in vivo: clinical significance of fibronectin and fibronectin receptor expressed in human glioma tissues. Clin Exp Metastasis. 1998; 16:729-741.

19. Ljubimova JY LA, Loksh A, Yong WH, Riedinger MS, Miner JH, Sorokin LM, Ljubimov AV, Black KL. Overexpression of alpha4 chain-containing laminins in human glial tumors identified by gene microarray analysis. Cancer Res. 2001; 61:5601-5610.

20. Nagato S NK, Harada H, Kohno S, Fujiwara H, Sekiguchi $\mathrm{K}$, Ohue S, Iwata S, Ohnishi T. Downregulation of laminin alpha4 chain expression inhibits glioma invasion in vitro and in vivo. Int J Cancer. 2005; 117:41-50.

21. Sengupta S NS, Hindi ES, Wainwright DA, Han Y, Lesniak MS. Short hairpin RNA-mediated fibronectin knockdown delays tumor growth in a mouse glioma model. Neoplasia. 2010; 12:837-847.

22. Samuels Y DLJ, Schmidt-Kittler O, Cummins JM, Delong L, Cheong I, Rago C, Huso DL, Lengauer C, Kinzler KW, 
Vogelstein B, Velculescu VE. Mutant PIK3CA promotes cell growth and invasion of human cancer cells. Cancer Cell. 2005; 7:561-573.

23. Jiménez C PR, Mellado M, Rodríguez-Frade JM, Collard J, Serrano A, Martínez-A C, Avila J, Carrera AC. Role of the $\mathrm{PI} 3 \mathrm{~K}$ regulatory subunit in the control of actin organization and cell migration. J Cell Biol. 2000; 151:249-262.

24. Zawel L. P3Ka: A Driver of Tumor Metastasis? Oncotarget. 2010; 1:315.

25. Yu J ZY, McIlroy J, Rordorf-Nikolic T, Orr GA, Backer JM. Regulation of the p85/p110 phosphatidylinositol 3'-kinase: stabilization and inhibition of the p110alpha catalytic subunit by the p85 regulatory subunit. Mol Cell Biol. 1998; 18:1379-1387.

26. Geering B CP, Nock G, Gharbi SI, Vanhaesebroeck B. Class IA phosphoinositide 3-kinases are obligate p85-p110 heterodimers. Proc Natl Acad Sci U S A. 2007; 104:78097814.

27. Kraus JA, Glesmann N, Beck M, Krex D, Klockgether T, Schackert G, Schlegel U. Molecular analysis of the PTEN, TP53 and CDKN2A tumor suppressor genes in long-term survivors of glioblastoma multiforme. Journal of neurooncology. 2000; 48:89-94.

28. Shukla S, MacLennan GT, Hartman DJ, Fu P, Resnick MI, Gupta S. Activation of PI3K Akt signaling pathway promotes prostate cancer cell invasion. International journal of cancer. 2007; 121:1424-1432.

29. Gallia G, Rand V, Siu I, Eberhart C, James C, Marie S, Oba-Shinjo S, Carlotti C, Caballero O, Simpson A, Brock M, Massion P, Carson BS, Riggins G. PIK3CA Gene Mutations in Pediatric and Adult Glioblastoma Multiforme. Mol Cancer Res. 2006; 4:709-714.

30. Konstantinova D, Kaneva R, Dimitrov R, Savov A, Ivanov S, Dyankova T, Kremensky I, Mitev V. Rare mutations in the PIK3CA gene contribute to aggressive endometrial cancer. DNA Cell Biol. 2010; 29:65-70.

31. Samuels Y, Wang Z, Bardelli A, Silliman N, Ptak J, Szabo S, Yan H, Gazdar A, Powell SM, Riggins GJ, Willson JK, Markowitz S, Kinzler KW, Vogelstein B, VE. V. High frequency of mutations of the PIK3CA gene in human cancers. Science. 2004; 304:554.

32. Gymnopoulos M, Elsliger MA, Vogt PK. Rare cancerspecific mutations in PIK3CA show gain of function. Proceedings of the National Academy of Sciences. 2007; 104:5569.

33. Rao SK EJ, Joshi AD, Siu IM, Riggins GJ. A survey of glioblastoma genomic amplifications and deletions. Journal of Neurooncology. 2010; 96:169-179.

34. Philp AJ CI, Leet C, Vincan E, Rockman SP, Whitehead RH, Thomas RJ, Phillips WA. The phosphatidylinositol 3'-kinase p85alpha gene is an oncogene in human ovarian and colon tumors. Cancer Res. 2001; 61:7426-7429.

35. Park SW KM, Eom HS, Han JY, Ahn CH, Kim SS, Lee $\mathrm{SH}$, Yoo NJ. Somatic mutation of PIK3R1 gene is rare in common human cancers. Acta Oncologica. 2010; 49:124127.

36. Urick ME, Rudd ML, Godwin AK, Sgroi D, Merino M, Bell DW. PIK3R1 (p85alpha) is somatically mutated at high frequency in primary endometrial cancer. Cancer Res. 2011; 71:4061-4067.

37. Jaiswal BS, Janakiraman V, Kljavin NM, Chaudhuri S, Stern HM, Wang W, Kan Z, Dbouk HA, Peters BA, Waring P, Dela Vega T, Kenski DM, Bowman KK, Lorenzo M, Li $\mathrm{H}$ WJ, Modrusan Z et al. Somatic mutations in p85alpha promote tumorigenesis through class IA PI3K activation. Cancer Cell. 2009; 16:463-474.

38. Sun M HP, Hofmann BT, Hart JR, Vogt PK. Cancerderived mutations in the regulatory subunit p85alpha of phosphoinositide 3-kinase function through the catalytic subunit p110alpha. Proc Natl Acad Sci U S A. 2010; 107:15547-15552.

39. Yu J WC, Backer JM. Regulation of the p85/p110alpha phosphatidylinositol 3'-kinase. Distinct roles for the n-terminal and c-terminal SH2 domains. J Biol Chem. 1998; 273:30199-30203.

40. Zhao L VP. Helical domain and kinase domain mutations in p110alpha of phosphatidylinositol 3-kinase induce gain of function by different mechanisms. Proc Natl Acad Sci U S A. 2008; 105:2652-2657.

41. Miled N, Yan Y, Hon WC, Perisic O, Zvelebil M, Inbar Y, Schneidman-Duhovny D, Wolfson HJ, Backer JM, RL W. Mechanism of two classes of cancer mutations in the phosphoinositide 3-kinase catalytic subunit. Science. 2007; 317:239-242.

42. Mizoguchi M NC, Mohapatra G, Louis DN. Genetic alterations of phosphoinositide 3-kinase subunit genes in human glioblastomas. Brain Pathol. 2004; 14:372-377.

43. Claudio J, Zhan F, Zhuang L, Khaja R, Zhu Y, Sivananthan K, Trudel S, Masih-Khan E, Fonseca R, Bergsagel P. Expression and mutation status of candidate kinases in multiple myeloma. Leukemia. 2007; 21:1124-1127.

44. Cordelières F. Manual Tracking (ImageJ). http://pacificmpicbgde/wiki/indexphp/Manual_Tracking. 2004.

45. Rasband WS: ImageJ, U. S. National Institutes of Health, Bethesda, Maryland, USA, http://imagej.nih.gov/ij/. In.; 1997-2011.

46. Kuan C, Wikstrand C, Bigner D. EGF mutant receptor vIII as a molecular target in cancer therapy. Endocrine-related cancer. 2001; 8:83.

47. Tchaicha JH, Reyes SB, Shin J, Hossain MG, Lang FF, McCarty JH. Glioblastoma angiogenesis and tumor cell invasiveness are differentially regulated by 8 integrin. Cancer research. 2011:Published OnlineFirst August 22, 2011.

48. van den Hoogen C, van der Horst G, Cheung H, Buijs JT, Pelger R, van der Pluijm G. Integrin [alpha] v Expression Is Required for the Acquisition of a Metastatic Stem/ Progenitor Cell Phenotype in Human Prostate Cancer. The 
American Journal of Pathology. 2011:Article in Press.

49. Matsunaga T, Takemoto N, Sato T, Takimoto R, Tanaka I, Fujimi A, Akiyama T, Kuroda H, Kawano Y, Kobune M, Kato J, Hirayama Y, Sakamaki S, Kohda K, Miyake $\mathrm{K}, \mathrm{Y}$ N. Interaction between leukemic-cell VLA-4 and stromal fibronectin is a decisive factor for minimal residual disease of acute myelogenous leukemia. Nature Med. 2003; 9:1158-1165.

50. Lightfoot HM Jr LA, Livasy CA, Moore DT, Cowan D, Dressler L, Craven RJ, Cance WG. Upregulation of focal adhesion kinase (FAK) expression in ductal carcinoma in situ (DCIS) is an early event in breast tumorigenesis. Breast Cancer Res Treat. 2004; 88:109-116.

51. Liu M, Yang Y, Wang C, Sun L, Mei C, Yao W, Liu Y, Shi Y, Qiu S, Fan J. The effect of epidermal growth factor receptor variant III on glioma cell migration by stimulating ERK phosphorylation through the focal adhesion kinase signaling pathway. Archives of biochemistry and biophysics. 2010; 502:89-95.

52. Fotiadou PP TC, Rajabi HN, Ewen ME. Wild-type NRas and KRas perform distinct functions during transformation. Mol Cell Biol. 2007; 27:6742-6755.

53. Chamberlain MD CT, Oberg JC, Hawrysh AD, James KM, Saxena A, Xiang J, Anderson DH. Disrupted RabGAP function of the p85 subunit of phosphatidylinositol 3-kinase results in cell transformation. J Biol Chem. 2008; 283:15861-15868.

54. Park SY LJ, Ha M, Nam JW, Kim VN. miR-29 miRNAs activate $\mathrm{p} 53$ by targeting $\mathrm{p} 85$ alpha and CDC42. Nat Struct Mol Biol. 2009; 16:23-29.

55. Senft J HB, Frisch SM. Caspase- 8 interacts with the $\mathrm{p} 85$ subunit of phosphatidylinositol 3-kinase to regulate cell adhesion and motility. Cancer Res. 2007; 67:11505-11509.

56. Gondi CS, Lakka SS, Dinh DH, Olivero WC, Gujrati M, Rao JS. Downregulation of UPA, UPAR and MMP-9 using small, interfering, hairpin RNA (siRNA) inhibits glioma cell invasion, angiogenesis and tumor growth. Neuron glia biology. 2004; 1:165.

57. Kargiotis O, Chetty C, Gondi CS, Tsung AJ, Dinh DH, Gujrati M, Lakka SS, Kyritsis A, Rao JS. Adenovirusmediated transfer of siRNA against MMP-2 mRNA results in impaired invasion and tumor-induced angiogenesis, induces apoptosis in vitro and inhibits tumor growth in vivo in glioblastoma. Oncogene. 2008; 27:4830.

58. Shingu T, Yamada K, Hara N, Moritake K, Osago H, Terashima M, Uemura T, Yamasaki T, M T. Growth inhibition of human malignant glioma cells induced by the PI3-K-specific inhibitor. J Neurosurg. 2003; 98:154-161.

59. Nakamura JL KA, Arvold ND, Gottschalk AR, Pieper RO, Stokoe D, Haas-Kogan DA. PKB/Akt mediates radiosensitization by the signaling inhibitor LY294002 in human malignant gliomas. J Neurooncol. 2005; 71:215222.

60. Su JD ML, Donner DB, Durden DL. PTEN and phosphatidylinositol 3'-kinase inhibitors up-regulate p53 and block tumor-induced angiogenesis: evidence for an effect on the tumor and endothelial compartment. Cancer Res. 2003; 63:3585-3592.

61. Schmidt-Kittler O, Zhu J, Yang J, Liu G, Hendricks W, Lengauer C, Gabelli SB, Kinzler KW, Vogelstein B, Huso DL. PI3Ka Inhibitors That Inhibit Metastasis. Oncotarget. 2010; 1:339.

62. Chagpar RB, Links PH, Pastor MC, Furber LA, Hawrysh $\mathrm{AD}$, Chamberlain MD, DH A. Direct positive regulation of PTEN by the p85 subunit of phosphatidylinositol 3-kinase. Proc Natl Acad Sci U S A. 2010; 107:5471-5476.

63. Hill KM HY, Yip SC, Yu J, Segall JE, Backer JM. $\mathrm{N}$-terminal domains of the class ia phosphoinositide 3-kinase regulatory subunit play a role in cytoskeletal but not mitogenic signaling. J Biol Chem. 2001; 276:1637416378 . 